\title{
Estimation of Message Source and Destination from Network Intercepts
}

\author{
Derek Justice* and Alfred Hero \\ Department of Electrical Engineering and Computer Science \\ University of Michigan \\ 1301 Beal Ave \\ Ann Arbor, MI 48109 \\ Email: justiced@umich.edu and hero@eecs.umich.edu
}

\begin{abstract}
We consider the problem of estimating the endpoints (source and destination) of a transmission in a network based on partial measurement of the transmission path. Sensors placed at various points within the network provide the basis for endpoint estimation by indicating that a specific transmission has been intercepted at their assigned locations. During a training phase, test transmissions are made between various pairs of endpoints in the network and the sensors they activate are noted. Sensor activations corresponding to transmissions with unknown endpoints are also observed in a monitoring phase. A semidefinite programming relaxation is used in conjunction with the measurements and linear prior information to produce likely sample topologies given the data. These samples are used to generate Monte Carlo approximations of the posterior distributions of source/destination pairs for measurements obtained in the monitoring phase. The posteriors allow for maximum a posteriori (MAP) estimation of the endpoints along with computation of some resolution measures. We illustrate the method using simulations of random topologies.
\end{abstract}

Index Terms-Network tomography and surveillance, detection and identification of anomalous events, channel and network models, data acquisition and sensor models

\section{INTRODUCTION}

We present a method to estimate the endpoints (source and destination) of a data transmission in a network whose logical topology is unknown. We assume there are a number of asynchronous sensors placed on some subset of elements (links or nodes) in a network. A sensor is activated, and its activation recorded, whenever the path of a data transmission is intercepted on the element where the sensor is situated. The measurement apparatus is illustrated on a sample network in Fig. 1. If multiple sensors are activated by a single transmission, they may not capable of providing the precise order in which they were activated. In general, a probability distribution on the possible orders of activation $P_{k}(\rho)$ is provided. For example, a transmission with endpoints $u_{1}=\left(\sigma_{1}, \delta_{1}\right)$ in Fig. 1 might activate $y_{1}=\left\{\gamma_{2}, \gamma_{3}\right\}$. The ordering $\left(\gamma_{2}, \gamma_{3}\right)$, corresponding to $\rho=1$, might have probability
$P(1)=\frac{3}{4}$, while the ordering $\left(\gamma_{3}, \gamma_{2}\right)$, where $\rho=2$, has probability $P(2)=\frac{1}{4}$. Since the orderings are defined over distinct sensor sets, we implicitly assume the transmission does not cycle in its path-that is, a particular sensor is activated at most once by a single transmission. During a preliminary training phase, the network is probed by transmitting data packets between various pairs of probing sites $\left\{u_{k}=\left(\sigma_{k}, \delta_{k}\right)\right\}_{k=1}^{K_{o}-1}$, and the sensors $\left\{y_{k}\right\}_{k=1}^{K_{o}-1}$ activated by each transmission are recorded. A monitoring phase also occurs, whereby we observe sensor activation sets $\left\{y_{k}\right\}_{k=K_{o}}^{K}$ for which the endpoints are unknown.

The resulting data $\left\{x_{k}\right\}_{k=1}^{K}=\left\{u_{k}, y_{k}\right\}_{k=1}^{K_{o}-1} \cup$ $\left\{y_{k}\right\}_{k=K_{o}}^{K}$ and ordering distributions $P_{k}(\rho)$ for $k=$ $1,2, \ldots, K$ along with some prior information about the network topology is processed by the system shown in Fig. 2 to produce Monte Carlo estimates of the posterior distributions of possible endpoints of those transmissions observed in the monitoring phase. We allow prior information of the form $Q(\bar{A})=b$ on the logical $(\{0,1\})$ adjacency matrix $A$ describing connections among sensors and probing sites. $\bar{A}$ is some subset of the elements of $A, Q$ is a linear operator, and $b$ is a vector. Thus the prior information is essentially a set of linear equalities that the adjacency matrix $A$ ought to satisfy. The linear operator $Q$ can be expressed as an equivalent matrix if the elements of $\bar{A}$ are organized in a vector $a$. The linear prior information is then of the form $Q a=b$. Given arbitrary $Q$ and $b$ the computation of feasible solutions to the linear equation is no small task, in fact it is known to be an NP-Complete problem [1]. We consider the associated minimum norm problem $\min \|Q a-b\|_{\Lambda}^{2}$ where $a \in\{0,1\}^{n}$ and $\|\cdot\|_{\Lambda}$ is a quadratic norm with respect to the positive definite matrix $\Lambda$. It is known that combinatorial optimization problems of this type may be successfully approximated by 'lifting' them into a higher dimensional matrix space where $X_{i j}=a_{i} a_{j}$ and $X \in\{0,1\}^{n \times n}[2]$.

With the advent of polynomial time interior point 


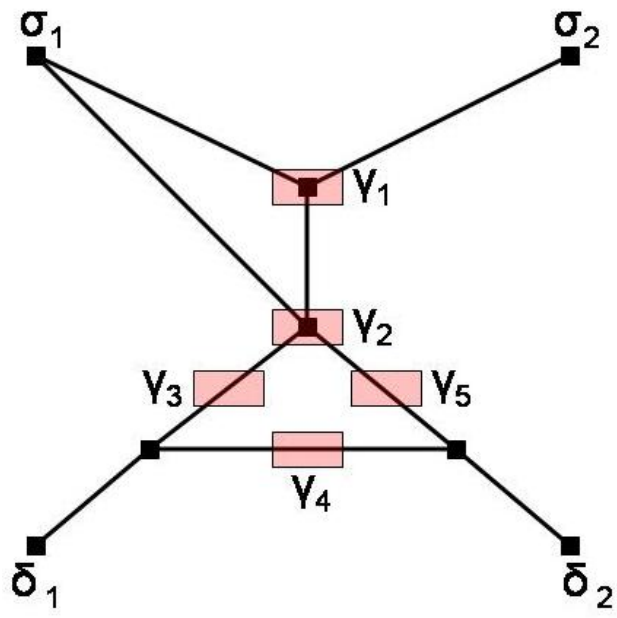

Fig. 1. Diagram of the measurement apparatus on a sample network. Probing sites are sources $\Sigma=\left\{\sigma_{1}, \sigma_{2}\right\}$ and destinations $\Delta=\left\{\delta_{1}, \delta_{2}\right\}$. A box on a link or node represents a sensor that indicates when a transmission path intercepts that link/node. We see $\gamma_{1}$ and $\gamma_{2}$ monitor nodes while $\gamma_{3}, \gamma_{4}$, and $\gamma_{5}$ monitor links.

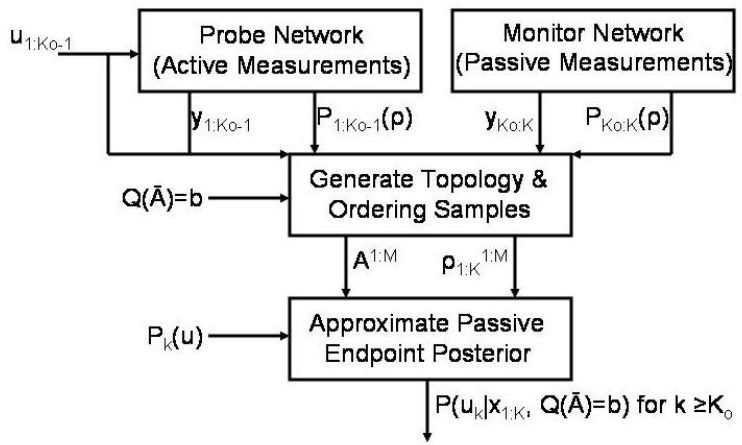

Fig. 2. Diagram of the transmission endpoint estimation system, assuming sensors have already been deployed.

methods for linear programming that can be extended to semidefinite programming [3], [4], it is convenient to consider a semidefinite programming (SDP) relaxation of the higher dimensional problem. Indeed, SDP relaxations have proven to be powerful tools for approximating hard combinatorial problems [5], [6], [7], [8]. The SDP, however, is solved over a continuous domain so it is necessary to retrieve a $0-1$ solution from the possibly fractional SDP solution. One possibility is a branch and bound scheme whereby certain variables are fixed and the SDP is repeated until a discrete solution is found [1], [8]. The branch and bound algorithm can take an exponential amount of time, depending on how tight the desired bound is. A randomized rounding scheme was developed in [6] for SDP relaxations of the maximum cut (MAXCUT) and maximum 2-satisfiability (MAX2SAT) problems. This scheme is shown to produce solutions of expected value at least 0.878 times the optimal value in [6]. We develop an SDP relaxation of the 01 minimum norm problem and apply the randomized rounding method in conjunction with samples from the ordering distributions $\left\{\rho_{1: K}^{m}\right\}_{m=1}^{M}$ to produce a number of network topology adjacency matrices $\left\{A^{m}\right\}_{m=1}^{M}$ that approximately satisfy the linear prior information $Q(\bar{A})=b$. We derive an expression for the expected value of the squared error $E\left[\|Q a-b\|_{\Lambda}^{2}\right]$ of samples produced in this way. This expression depends on the solution of the SDP relaxation, but an upper bound on the error independent of the SDP solution is also given.

We wish to produce posterior distributions given the data and prior information of the endpoints of transmissions observed in the monitoring phase $P\left(u_{\hat{k}} \mid x_{1: K}, Q(\bar{A})=b\right)$ for $\hat{k} \geq K_{o}$. The network topology and sensor ordering samples are used in conjunction with prior distributions on the endpoints of measurements made during the monitoring phase $P_{k}(u)$ for $k=K_{o}, K_{o}+1, \ldots, K$ to compute Monte Carlo approximations of the desired posterior distributions via Bayes rule. Bayes formula for this problem essentially 
reduces to the expected value of a functional of the topology $A$ and sensor ordering $\rho$; our approximation of the endpoint posterior thus becomes an average of the values of this functional at each sample topology $A^{m}$ and ordering set $\rho_{1: K}^{m}$. It is readily apparent that this functional requires the conditionals $P(y \mid u, \rho, A)$-these path likelihood functions are the conditional probabilities of a sensor activation set $y$ given the endpoints $u$ and activation order $\rho$ in a topology $A$. We propose a path likelihood model inspired by shortest path routing, whereby the length of a path determines its probability. With the endpoint posterior distribution in hand, we can immediately give the MAP estimate of $u_{\hat{k}}$ or an a posteriori confidence region of probable source/destination pairs.

The related area of network tomography has recently been a subject of substantial research. It refers to the use of traffic measurements over parts of a network to infer characteristics of the complete network. Some characteristics of interest include the following: source/destination traffic rates [9], [10], link-level packet delay distributions [11], [12], link loss [13], and link topology [14], [15]. For an overview of relevant tomography problems for the Internet see [16]. In many applications, the tomography problem is ill posed since data is insufficient to determine a unique topology or delay distribution.

Our work is related to the internally sensed network tomography application described in [17], [18]. These works propose a methodology for estimating the topology of a telephone network using the measurement apparatus illustrated in Fig. 1. The data transmissions are of course telephone calls and the asynchronous sensors are located on trunk lines. A simple argument in [18] demonstrates that the number of topologies consistent with the data measured during the probing phase $\left\{x_{k}\right\}_{k=1}^{K_{o}-1}$ is exponential in the number of sensors. Indeed the problem is ill-posed as the data required to provide a reasonable estimate of the topology will never be available in practice. We sidestep the difficulties of developing a single topology estimate by averaging over many probable topologies in computing the endpoint posterior distribution.

The solution approach we develop is very general, and we suspect it might have application in all sorts of networks: including telephone networks as described in [17], the Internet, social networks (such as command and control structures), or biological networks (such as protein-protein interaction networks) [19], [20]. Since we allow for sensor placement on arbitrary network elements, the method is equally applicable to networks where it may be more convenient to monitor nodes (as in the Internet) or monitor links (as in the telephone network of [17]). Also, the ordering distributions allow for situations involving sensors ranging from asynchronous to perfectly synchronized. At one extreme, the sensors are exactly synchronized-in which case the distribution $P_{k}(\rho)$ reduces to a delta function with all mass concentrated on the known ordering of sensors. In a large network with imperfect synchronization, a natural source of such information would be the geographical locations of the sensors-although we do not know the logical topology of the network of interest, we do know geographically where the sensors were placed. For example, if we had sensors scattered across a telephone network that spanned the United States, any ordering $\rho$ that implied an adjacency between a sensor in Los Angeles and one in New York would be highly unlikely. When the network of interest is the Internet with sensors placed on routers, the 'traceroute' command might be used to obtain the ordering distributions. Suppose traceroute is enabled on some routers and disabled on others; one could then assign zero probability to all orderings of a transmission that are not consistent with the traceroute path. There is a tremendous amount of flexibility.

Although the monitored network topology is unknown, the linear prior information permits inclusion of reasonably available information relevant to the topology. This is a generalization of the frequently used vertex degree prior. Vertex degree priors are used quite often due to the fact that many real world networks are characterized by specific degree distributions [21]. For example, studies have suggested a power-law distribution describes vertex degrees in the Internet [22]. Such priors have recently been applied to research involving models of social and biological networks [19], [20], [23]. Since the degree of a vertex is equal to the sum over the row of the adjacency matrix describing connections to that vertex, one can easily construct a linear operator $Q$ so that $Q(\bar{A})=b$ expresses the degree prior for a given vector of vertex degrees $b$.

The approach described here might also find utility in systems conveniently modelled by graphs, such as finite state automata. The problem of machine identification is a classic problem in the theory of automata testing [24], [25]. Here, we are given a black box with an automaton inside whose transition function is unknown. Based on the response of the system to certain input sequences, we wish to reconstruct the transition function. The link to the network topology recovery aspect of our problem is clear, since a graph provides a convenient representation for the transition function of interest. The probing sites chosen in the probing phase of our problem is analogous to the input sequences to the black box automaton. Similarly, link sensors correspond to events in the automaton's observable event set. An exhaustive algorithm for solving this problem is given in [24] and shown to have exponential run time. Our methods might be adapted to provide a polynomial time approximation 
algorithm. This would involve partitioning measurements with cycles (whereby an observable event occurs more than once in the same string) to satisfy the direct path assumption and selecting a different conditional path likelihood $P(y \mid u, \rho, A)$ since the shortest path routing model we suggest might not be appropriate.

The outline of this paper is as follows. We review the problem, describe in detail each component of the endpoint estimation system (Fig. 2), and analyze its complexity in Section II. In Section III, we provide some simulations of random graphs. In Section IV we conclude with some extensions of the method presented here and give directions for future work utilizing feedback for adaptive probing.

\section{Model AND Theory For Source-Destination ESTIMATION}

Let $G(V, E, f)$ be a simple graph defined by the vertex set $V$, edge set $E$, and incidence relation $f: E \rightarrow V \times V$ giving the vertices connected by each edge. We allow $G$ to be either directed or undirected; however, it should be known a priori which is the case. In our application, $E$ defines the set of links in the network topology, $V$ defines the routers or switches connected by these links, and $f$ determines the pair of routers/switches connected by each link. The graph $G$ is unknown to us.

Let $\Gamma$ denote a set of sensors we place in the network. Sensors are placed on some subset of graph elements; that is sensors may be placed on vertices, edges, or both. A sensor will indicate whenever a transmission through the network passes the element it is monitoring. Probing sites are selected from the vertex set $V$. The source vertex set $\Sigma \subseteq V$ is the set of vertices from which transmissions may originate, and the destination vertex set $\Delta \subseteq V$ are those vertices at which transmissions may terminate. A path between probing sites $s_{k} \in \Sigma$ and $d_{k} \in \Delta$ is given by $y_{k} \subseteq \Gamma$, where $y_{k}$ contains the sensors activated by the transmission from $s_{k}$ to $d_{k}$. Because the sensors are in general asynchronous, the paths are unordered sets. However, along with each $y_{k}$, a discrete probability distribution $P_{k}(\rho)$ is given on possible orderings $\rho$ of the set $y_{k}$. We assume a transmission does not cycle in its path from source to destination, so that only orderings of distinct elements of $y_{k}$ are considered. It follows that if $y_{k}$ has $\left|y_{k}\right|$ distinct elements, then $P_{k}(\rho)$ is defined over $\left|y_{k}\right|$ ! different orderings. Note that the case of perfectly synchronized sensors is easily handled in this framework: simply take $P_{k}(\rho)=\delta\left(\rho-\rho_{k}\right)$ where $\rho_{k}$ is the known order in which the sensors $y_{k}$ were activated.

The purpose of our system is to estimate the source and destination $u_{\hat{k}}=\left(s_{\hat{k}}, d_{\hat{k}}\right)$ of an activated sensor set $y_{\hat{k}}$ corresponding to a measurement whose endpoints are unknown (i.e. passive measurement). In order to estimate the endpoints of such a measurement, it is necessary to have some idea of the logical topology of the network. Instead of considering the logical adjacencies implied by the actual network $G(V, E, f)$, we are concerned with adjacency relationships among only those elements (vertices and edges) that are either monitored with a sensor or used as a probing site. For example, we cannot hope to pinpoint the position of a link $e$ in the original network that is not monitored by a sensor. We assume unmonitored elements are essentially 'shortcircuited' in the original network $G$. The idea here is to assure two elements are logically adjacent even if they are physically separated by an element (or subgraph of elements) that is not monitored. The particular topology we consider is then $G_{A}\left(V_{A}, E_{A}\right)$ where $V_{A}=\Gamma \cup \Sigma \cup \Delta$ is the set of sensors and probing sites, and $E_{A} \subseteq V_{A} \times V_{A}$ describes the logical adjacencies among these elements. $G_{A}$ may be undirected or directed depending upon the nature of the network $G$. For computational purposes, we represent $G_{A}$ by its adjacency matrix $A$ where $A_{i j}=1$ if and only if $(i, j) \in E_{A}$ for $i, j \in V_{A}$ and $A_{i j}=0$ otherwise. An example logical topology $G_{A}$ is given in Fig. 3 for the monitored network $G$ in Fig. 1 .

We assume independence of measurements (that is, independence over the index $k$ ) and utilize a Bayesian framework to produce suitable approximations of the endpoint posterior distribution:

$$
\begin{aligned}
& P\left(u_{\hat{k}} \mid x_{1: K}, Q(\bar{A})=b\right)= \\
& E_{A, \rho_{1: K}}\left[\frac{P\left(y_{\hat{k}} \mid u_{\hat{k}}, \rho_{\hat{k}}, A\right) P_{\hat{k}}\left(u_{\hat{k}}\right)}{\sum_{u} P\left(y_{\hat{k}} \mid u, \rho_{\hat{k}}, A\right) P_{\hat{k}}(u)} \mid x_{1: K}, Q(\bar{A})=b\right]
\end{aligned}
$$

We have available linear prior information on some of the logical adjacency elements $\bar{A} \subseteq A$ of the form $Q(\bar{A})=b$ where $Q$ is a linear operator and a prior distribution on endpoints $P_{\hat{k}}(u)$. It is assumed the endpoint pair of a passive measurement is independent of the particular topology $A$, in other words, the parties communicating do not know the network topology either. However, if there is no connection between a given endpoint pair $u$ in a topology $A$, one would expect such a pair to have probability zero; we shall use a model for the term multiplying $P_{\hat{k}}(u)$ to ensure the product is zero in this case. Here $x_{1: K} \equiv\left\{x_{k}\right\}_{k=1}^{K}$ represents all measured data $\left(x_{k}=\left(u_{k}, y_{k}\right)\right.$ if the endpoints of measurement $k$, $u_{k}$, are known, otherwise $x_{k}=y_{k}$ ), and $\rho_{k}$ is the random ordering of the sensors activated in measurement $y_{k}$. The conditional expectation is therefore taken over all logical adjacency matrices $A$ and sensor orderings for all measurements $\rho_{1: K}$. We introduce a shortest path routing model for the conditional path probabilities $P(y \mid u, \rho, A)$. The conditional expectation in Eq. (1) is approximated in a Monte Carlo fashion by summing over the argument evaluated at a number of adjacency matrix and sensor ordering samples. The sensor orderings $\rho_{1: K}$ are drawn 


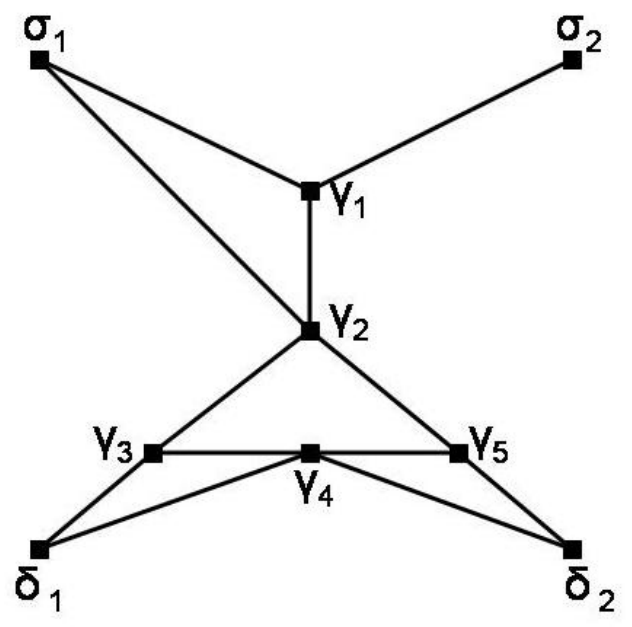

Fig. 3. Example logical topology $G_{A}\left(V_{A}, E_{A}\right)$ for the monitored network $G$ in Fig. 1. The vertex set of $G_{A}$ consists of sensors $\Gamma=\left\{\gamma_{i}\right\}_{i=1}^{5}$ and probing sites $\Sigma=\left\{\sigma_{1}, \sigma_{2}\right\}, \Delta=\left\{\delta_{1}, \delta_{2}\right\}$, so that $V_{A}=\Gamma \cup \Sigma \cup \Delta$. The edges of $G_{A}$ summarize logical adjacencies among sensors and probing sites with any intervening unmonitored elements short-circuited.

independently from available distributions $P_{k}(\rho)$ for $k=1,2, \ldots, K$. These are used in conjunction with the solution to a semidefinite programming relaxation that incorporates the prior information $Q(\bar{A})=b$ to produce adjacency matrix samples $A$ that are likely given both the data and the prior information. With the approximate endpoint posterior distribution in hand, we can provide MAP estimates of the endpoints of the passive measurement and compute appropriate error measures.

In the following, we first elaborate on probing of the network and the characterization of measurements obtained. Then we describe the distribution $P\left(A, \rho_{1: K} \mid x_{1: K}, Q(\bar{A})=b\right)$ and how it may be efficiently sampled using the given ordering distributions and a semidefinite programming relaxation. Next we discuss how the samples are used to approximate the endpoint posterior and produce MAP estimates. Finally, we analyze the complexity of our algorithm.

\section{A. Probing the Network and Taking Measurements}

The set of all available measurements $\left\{x_{k}\right\}_{k=1}^{K}$ is partitioned into two disjoint sets. The measurements for $k=1,2, \ldots K_{o}-1$ correspond to a training phase for the probing sites $\Sigma, \Delta$. For each $k<K_{o}$, we select a probing pair $u_{k} \in \Sigma \times \Delta$ and pass a transmission between this pair to observe the sensors $y_{k}$ activated. The measurement data therefore consists of both the endpoints and the activated sensor set $x_{k}=\left(u_{k}, y_{k}\right)$ for $k<K_{o}$. Such a measurement is referred to as an active measurement. The remaining measurements provide only an activated sensor set: $x_{k}=y_{k}$ for $K_{o} \leq k \leq K$. These are referred to as passive measurements since the endpoints of the associated transmission are unknown. It is assumed, however, that the endpoints are realizations of a random probing site pair described by the known distribution $P_{k}(u)$ defined on $\Sigma \times \Delta$. We desire to estimate the particular probing site pair between which a transmission was passed resulting in a given passive measurement. Along with the unordered set of activated sensors $y_{k}$ we are given a distribution on the order in which the sensors were activated $P_{k}(\rho)$ for every $k=1,2, \ldots K$ (both active and passive measurements). The distributions on ordering come from some absolute prior information we have about our sensors-such as geography or 'traceroute' paths as discussed earlier. We will make some independence assumptions about ordering in the following section.

\section{B. Generating Topology and Sensor Ordering Samples}

In order to produce a Monte Carlo estimate of the conditional expectation in Eq. (1), we need to specify and sample from the distribution $P\left(A, \rho_{1: K} \mid x_{1: K}, Q(\bar{A})=\right.$ $b)$. We first expand this distribution as

$$
\begin{aligned}
& P\left(A, \rho_{1: K} \mid x_{1: K}, Q(\bar{A})=b\right)= \\
& P\left(A \mid x_{1: K}, \rho_{1: K}, Q(\bar{A})=b\right) P\left(\rho_{1: K} \mid Q(\bar{A})=b\right)
\end{aligned}
$$

where the ordering variables $\rho_{1: K}$ are assumed independent of the particular configurations of sensors activated $x_{1: K}$. As described previously, the distributions over orderings are determined by some relatively certain prior information, such as geography. The activated sensor set simply serves to specify the domain of the ordering distribution; for example, if there are three elements in $y_{k}$, then the distribution $P_{k}(\rho)$ is defined over $3 !=6$ distinct orderings. 
An additional independence assumption is made to simplify the expression in Eq. (2): specifically, we assume $P\left(\rho_{1: K} \mid Q(\bar{A})=b\right)=P\left(\rho_{1: K}\right)$. This is similar to a factorization assumption made in variational EM algorithms [26]. $\bar{A}$ can be treated as a parameter set and $\rho_{1: K}$ can be treated as hidden variables. The assumption in variational EM is to consider a factored distribution over these random variables $q\left(\bar{A}, \rho_{1: K}\right)=q_{A}(\bar{A}) q_{\rho}\left(\rho_{1: K}\right)-$ an independence assumption. We will further justify this approximation later by performing some pre-processing on the set $\bar{A}$ to remove adjacency elements that are likely to be dependent on some ordering variable $\rho_{k}$. Combining this with the independence over measurements assumption, we then have

$$
\begin{aligned}
& P\left(A, \rho_{1: K} \mid x_{1: K}, Q(\bar{A})=b\right)= \\
& P\left(A \mid x_{1: K}, \rho_{1: K}, Q(\bar{A})=b\right) \prod_{k=1}^{K} P_{k}\left(\rho_{k}\right)
\end{aligned}
$$

where $P_{k}\left(\rho_{k}\right)$ are known distributions over the ordering of sensors in measurement $k$. The factored form of the distribution in Eq. (3) suggests the first thing we should do in generating our samples is to select orderings $\rho_{k}$ independently from the distributions $P_{k}$ for each $k=$ $1,2, \ldots, K$. This is a simple matter since each $P_{k}$ is a discrete distribution defined over a finite number of orderings.

Consider now what a measurement $x_{k}$ equipped with an ordering $\rho_{k}$ implies about the adjacency matrix $A$. Let $x_{k \rho_{k}}$ denote the ordered measurement where, if $x_{k}$ is an active measurement, the source probing site is taken as the first element followed by the ordering $\rho_{k}$ of the activated sensors and the destination probing site is taken as the last element. If $x_{k}$ is a passive measurement, $x_{k \rho_{k}}$ is simply the ordering $\rho_{k}$ of the activated sensors. The fact that the transmission passes from the $l^{\text {th }}$ element of $x_{k \rho_{k}}$, given by $x_{k \rho_{k}}^{l}$, to $x_{k \rho_{k}}^{l+1}$ implies there must be a logical connection between $x_{k \rho_{k}}^{l}$ and $x_{k \rho_{k}}^{l+1}$. Thus if we select an ordering $\rho_{k}$ for each measurement (i.e. for $k=1,2, \ldots K)$, then every adjacency element in the set $A^{x \rho}$ must be 1 , where $A^{x \rho}$ is defined by

$$
A^{x \rho}=\left\{A_{i j} \mid \exists k, l:\left(x_{k \rho_{k}}^{l}, x_{k \rho_{k}}^{l+1}\right)=(i, j)\right\}
$$

Once we draw orderings $\rho_{1: K}$ as previously described, the adjacency matrix elements in $A^{x \rho}$ are immediately fixed at unity by these. It remains, however, to select the remaining adjacency elements. In drawing these, we must account for the prior information $Q(\bar{A})=b$. Since $Q$ is a linear operator, we may re-express this information as $Q a=b$ where $Q$ is now understood to be a matrix and $a \in\{0,1\}^{n}$ is a vectorized version of the adjacency elements $\bar{A}$. For arbitrary $Q$, finding a $0-1$ vector $a$ that satisfies the equation $Q a=b$ is an NPComplete problem [27]. We will shortly discuss how randomized rounding of a semidefinite programming relaxation may be used to find approximate solutions. The randomized rounding will induce a distribution on $P\left(A \mid x_{1: K}, \rho_{1: K}, Q(\bar{A})=b\right)$, the remaining factor in Eq. (3). The induced distribution will have the desirable property that it assigns high probability to samples that approximately satisfy the linear constraint $Q(\bar{A})=b$.

Consider the matrix equation $Q a=b$ equivalent to the linear prior information $Q(\bar{A})=b$. Producing vectors $a$ that satisfy this equation amounts to finding several solutions to the problem

$$
\begin{aligned}
& \text { find } a \in\{0,1\}^{n} \\
& \text { such that } Q a=b
\end{aligned}
$$

Unfortunately, the problem in Eq. (5) is NP-complete [27]. We consider an equivalent restatement of Eq. (5)

$$
\begin{aligned}
& \text { minimize }(Q a-b)^{T} \Lambda(Q a-b) \\
& \text { such that } a \in\{0,1\}^{n}
\end{aligned}
$$

where $\Lambda$ is a (symmetric) positive definite matrix that may be chosen to emphasize the relative importance of the different constraints. Obviously any optimal solution of the problem in Eq. (6) with zero value solves the feasibility problem in Eq. (5). The problem in Eq. (6) is no easier than the original statement, however, it has been shown that problems of this type (0-1 quadratic programs) can be approximated quite well using a semidefinite relaxation [7].

We now proceed to derive the SDP relaxation of Eq. (6). Our relaxation is similar to the one derived in [6] for MAX2SAT. First note that the optimization in Eq. (6) is equivalent to

$$
\begin{aligned}
& \text { minimize } a^{T} D a-2 d^{T} a \\
& \text { such that } a \in\{0,1\}^{n}
\end{aligned}
$$

where $D=Q^{T} \Lambda Q$ and $d=Q^{T} \Lambda b$. This is easily seen by expanding the objective in Eq. (6) and dropping the constant term. Now note that $a_{i}^{2}=a_{i}$ since $a_{i} \in\{0,1\}$; this fact this allows Eq. (7) to be re-expressed as

$$
\begin{aligned}
& \text { minimize } \sum_{i, j} D_{i j} a_{i} a_{j}-2 \sum_{j} d_{j} a_{j}^{2} \\
& \text { such that } a \in\{0,1\}^{n}
\end{aligned}
$$

We now introduce variables $w_{i} \in\{-1,1\}$ for each $a_{i} \in$ $\{0,1\}$ for $i=1 \ldots n$ along with an additional $w_{n+1} \in$ $\{-1,1\}$ so that the change of variables is given by

$$
a_{i}=\frac{1}{2}\left(1+w_{n+1} w_{i}\right)
$$

The identities in Eq. (10) follow from this change of variables.

$$
\begin{aligned}
& a_{i} a_{j}= \\
& \frac{1}{4}\left[\left(1+w_{i} w_{j}\right)+\left(1+w_{n+1} w_{i}\right)+\left(1+w_{n+1} w_{j}\right)-2\right] \\
& -a_{i} a_{j}= \\
& \frac{1}{4}\left[\left(1-w_{i} w_{j}\right)+\left(1-w_{n+1} w_{i}\right)+\left(1-w_{n+1} w_{j}\right)-4\right]
\end{aligned}
$$


If we introduce a negative sign in the objective, then the optimization in Eq. (8) becomes

$$
\begin{aligned}
& \max \frac{1}{4} \sum_{i, j}\left[B_{i j}\left(1+w_{i} w_{j}\right)+C_{i j}\left(1-w_{i} w_{j}\right)-4 D_{i j}\right] \\
& \text { such that } w \in\{-1,1\}^{n+1}
\end{aligned}
$$

where $e$ is a vector of ones and matrices $B, C$ are given by

$$
\left.\begin{array}{rl}
B & =\left(\begin{array}{cc}
0 & 2 d \\
2 d^{T} & 0
\end{array}\right) \\
D & D e \\
(D e)^{T} & 0
\end{array}\right)
$$

In order to obtain a semidefinite program, define the matrix $W=w w^{T}$. It is simple to show that $W=w w^{T}$ for some vector $w$ if and only if $W \succeq 0$ (i.e. $W$ is positive semidefinite) and $\operatorname{rank}(W)=1$. We drop the nonconvex rank-1 constraint to obtain the SDP relaxation

$$
\begin{array}{ll}
\text { maximize } & \operatorname{Tr}[(B-C) W] \\
\text { such that } & \operatorname{diag}(W)=e \\
& W \succeq 0
\end{array}
$$

where $\operatorname{Tr}[\cdot]$ indicates the trace operation and the constraint $\operatorname{diag}(W)=e$ is added to enforce $w_{i}^{2}=1$. The equivalence of the objective functions in Eq. (13) and Eq. (11) can be seen easily by replacing $w_{i} w_{j}$ with $W_{i j}$ and dropping constant terms. The SDP in Eq. (13) may be solved in polynomial time using a primal-dual path following algorithm [4]. The result of this optimization $W^{*}$ will in general be a non-integer symmetric positive semidefinite matrix. In [6], a randomized rounding methodology is proposed to recover a $-1,1$ vector $w$ from the SDP solution $W^{*}$. The strategy is to first perform the Cholesky factorization $W^{*}=V^{T} V$. A random hyperplane through the origin with normal vector $r$ is then chosen by selecting $r$ from the uniform distribution on the surface of the unit hypersphere $S_{n}=\{r \in$ $\left.\mathbf{R}^{n+1} \mid r^{T} r=1\right\}$. The value of $w_{i}$ is then determined by whether the corresponding column $v_{i}$ of $V$ lies above or below the hyperplane, i.e. $w_{i}=1$ if $v_{i}^{T} r \geq 0$ and $w_{i}=-1$ if $v_{i}^{T} r<0$. The $i^{t h}$ element of the vectorized adjacency sample $\hat{a}$ is then given by

$$
\hat{a}_{i}= \begin{cases}1 & \text { if } \operatorname{sign}\left(v_{i}^{T} r\right)=\operatorname{sign}\left(v_{n+1}^{T} r\right) \\ 0 & \text { if } \operatorname{sign}\left(v_{i}^{T} r\right) \neq \operatorname{sign}\left(v_{n+1}^{T} r\right)\end{cases}
$$

This result can be seen by applying the rounding method and then using the change of variable formula given in Eq. (9).

We now proceed to derive the mean squared error $E\left[\|Q \hat{a}-b\|_{\Lambda}^{2}\right]$ of the sample adjacency in Eq. (14) and thereby quantify how close the samples produced in this way come to satisfying the linear prior information on average. First note that the rounding scheme used implies the following identities.

$$
\begin{aligned}
& E\left[1+w_{i} w_{j}\right]=2 P\left(\operatorname{sign}\left(v_{i}^{T} r\right)=\operatorname{sign}\left(v_{j}^{T} r\right)\right) \\
& E\left[1-w_{i} w_{j}\right]=2 P\left(\operatorname{sign}\left(v_{i}^{T} r\right) \neq \operatorname{sign}\left(v_{j}^{T} r\right)\right)
\end{aligned}
$$

where $r$ is a random vector from the uniform distribution on $S_{n}$ as previously defined. We may evaluate the probabilities in Eq. (15) quite easily via the observation in [6]. Note that symmetry of the distribution implies $P\left(\operatorname{sign}\left(v_{i}^{T} r\right) \neq \operatorname{sign}\left(v_{j}^{T} r\right)\right)=$ $2 P\left(v_{i}^{T} r \geq 0, v_{j}^{T} r<0\right)$. And if $\theta=\arccos \left(v_{i}^{T} v_{j}\right)$ is the angle between the vectors $v_{i}$ and $v_{j}$ then it follows $P\left(v_{i}^{T} r \geq 0, v_{j}^{T} r<0\right)=\frac{\theta}{2 \pi}$ since the distribution of $r$ is uniform on $S_{n}$. A similar argument applies to the case of matching sign. The results are summarized below.

$$
\begin{aligned}
& P\left(\operatorname{sign}\left(v_{i}^{T} r\right)=\operatorname{sign}\left(v_{j}^{T} r\right)\right)=1-\frac{1}{\pi} \arccos \left(v_{i}^{T} v_{j}\right) \\
& P\left(\operatorname{sign}\left(v_{i}^{T} r\right) \neq \operatorname{sign}\left(v_{j}^{T} r\right)\right)=\frac{1}{\pi} \arccos \left(v_{i}^{T} v_{j}\right)
\end{aligned}
$$

If we define the matrix $Z$ such that $Z_{i j}=\arccos \left(W_{i j}^{*}\right)$ where $W^{*}$ is the solution of the SDP relaxation in Eq. (13) and note that the objective function in Eq. (11) is exactly equal to $b^{T} \Lambda b-\|Q \hat{a}-b\|_{\Lambda}^{2}$, then we may take the expectation of the objective in Eq. (11) and apply the identities in Eqs. (15) and (16) to obtain the mean squared error as

$$
E\left[\|Q \hat{a}-b\|_{\Lambda}^{2}\right]=\|Q e-b\|_{\Lambda}^{2}-\frac{1}{2 \pi} \operatorname{Tr}[(C-B) Z]
$$

where $e$ is a vector of ones.

We may obtain a bound on the expected value of the squared error in Eq. (17) independent of the solution to the SDP. As in [6], define the constant $\alpha$

$$
\alpha=\min _{z \in[0, \pi]} \frac{2}{\pi} \frac{z}{1-\cos z}
$$

From this definition of $\alpha$, the following identities follow immediately

$$
\begin{aligned}
\frac{1}{2} \alpha(1+\cos z) & \leq 1-\frac{1}{\pi} z \\
\frac{1}{2} \alpha(1-\cos z) & \leq \frac{1}{\pi} z
\end{aligned}
$$

We take the expected value of the objective function in Eq. (11) and apply the identities in Eq. (19) with $Z_{i j}=$ $\arccos \left(W_{i j}^{*}\right)$ to give

$$
\begin{aligned}
& b^{T} \Lambda b-E\left[\|Q \hat{a}-b\|_{\Lambda}^{2}\right] \geq \\
& \alpha \frac{1}{4}\left(\sum_{i, j}\left[B_{i j}+C_{i j}\right]+\operatorname{Tr}\left[(B-C) W^{*}\right]\right)-e^{T} D e
\end{aligned}
$$

Now suppose the equation $Q a=b$ has at least one feasible solution $a^{0}$. Let $w^{0}$ be the corresponding $-1,1$ vector and $W^{0}=w^{0}\left(w^{0}\right)^{T}$. We then have

$$
\begin{aligned}
& 0=\left\|Q a^{0}-b\right\|_{\Lambda}^{2}=e^{T} D e+b^{T} \Lambda b- \\
& \frac{1}{4}\left(\sum_{i, j}\left[B_{i j}+C_{i j}\right]+\operatorname{Tr}\left[(B-C) W^{0}\right]\right)
\end{aligned}
$$


But since $W^{*}$ solves the SDP in Eq. (13), it follows

$$
\begin{aligned}
& \operatorname{Tr}\left[(B-C) W^{*}\right] \geq \operatorname{Tr}\left[(B-C) W^{0}\right]= \\
& 4 e^{T} D e+4 b^{T} \Lambda b-\sum_{i, j}\left[B_{i j}+C_{i j}\right]
\end{aligned}
$$

We may now combine the inequalities in Eqs. (20) and (22) and rearrange to obtain a bound on the expected value of the squared error that is independent of the SDP solution

$$
E\left[\|Q \hat{a}-b\|_{\Lambda}^{2}\right] \leq(1-\alpha)\left(\|Q e\|_{\Lambda}^{2}+\|b\|_{\Lambda}^{2}\right)
$$

In practice, the bound in Eq. (23) tends to exceed the true expected value in Eq. (17) by a large amount. However, it is of theoretical interest since it gives a general idea of how close samples produced in this way will come to satisfying the linear prior information, given the matrix $Q$ and vector $b$ specifying this information. One must be careful to apply this bound only when all elements of $Q$ and $b$ are nonnegative (such as when a vertex degree prior is used). A similar bound can be derived when some elements of $Q$ or $b$ are negative, but we will omit it here.

A naive procedure for generating the necessary samples using these procedures would be to first draw the ordering variables $\rho_{1: K}$ then fix the adjacency elements in $A^{x \rho}$ corresponding to the draw. One could then reduce the system $Q(\bar{A})=b$ by eliminating elements in $\bar{A} \cap A^{x \rho}$ and proceed to formulate and solve the SDP for use in randomized rounding. This approach is computationally prohibitive, however, because it requires solving a new SDP for every single sample. Instead, we prefer to solve a single SDP and use its solution to generate all samples. The single SDP is derived from the system $Q(\bar{A})=b$ where the eliminated variables $A_{i j}$ are those whose probability of being in the set $A^{x \rho}$ exceeds a threshold. The probability $P\left(A_{i j} \in A^{x \rho}\right)$ is computed from the ordering distributions $P_{k}(\rho)$ as

$$
P\left(A_{i j} \in A^{x \rho}\right)=\max _{k} \sum_{\rho \mid \exists l: x_{k \rho}^{l}=i, x_{k \rho}^{l+1}=j} P_{k}(\rho)
$$

Note that by fixing the variables that are likely to be in $A^{x \rho}$ and eliminating them from the prior constraints $Q(\bar{A})=b$, we are removing elements from $\bar{A}$ that are likely to depend on ordering variables $\rho$. This sort of decoupling serves to strengthen the validity of the independence assumption made earlier $P\left(\rho_{1: K} \mid Q(\bar{A})=\right.$ b) $=P\left(\rho_{1: K}\right)$.

There may be adjacency matrix elements that are not in $\bar{A}$ and have zero probability of being in $A^{x \rho}$. Define $A^{o} \equiv\left\{A_{i j} \mid A_{i j} \notin \bar{A}, P\left(A_{i j} \in A^{x \rho}\right)=0\right\} ; A^{o}$ then denotes the adjacency matrix elements that we have no information about. We adopt the principle of parsimony and assume all elements in $A^{o}$ are zero. A summary of our procedure for generating $M$ sample adjacency matrices and orderings follows.

- Compute $P\left(A_{i j} \in A^{x \rho}\right)$ for all $A_{i j} \in \bar{A}$ as in Eq. (24).

- Eliminate $\left\{A_{i j} \mid P\left(A_{i j} \in A^{x \rho}\right) \geq \delta\right\}$ from $\bar{A}$ and adjust the system $Q(\bar{A})=b$ with these variables fixed at 1.

- Solve the SDP corresponding to $Q(\bar{A})=b$ for the optimum $W^{*}$.

- Compute and store the Cholesky factor $V$ of the SDP solution $W^{*}$.

- For $m=1,2, \ldots M$

- Draw $\rho_{k}$ from $P_{k}(\rho)$ for $k=1,2, \ldots, K$.

- Determine $A^{x \rho}$ as in Eq. (4) and set $A_{i j}=1$ for all $A_{i j} \in A^{x \rho}$.

- Draw $r$ from the uniform distribution on $S_{n}$.

- Take inner products of the Cholesky factors with $r$ to determine $A_{i j} \notin A^{x \rho}$ that are organized in the vector $a$ as shown in Eq. (14).

- Set all remaining adjacency elements to 0 .

We may now write down the conditional distribution $P\left(A \mid x_{1: K}, \rho_{1: K}, Q(\bar{A})=b\right)$ from which the SDP rounding method is sampling. First define the set $H\left(A_{i j}\right)$ as

$$
\begin{aligned}
& H\left(A_{i j}\right)= \\
& \begin{cases}\left\{r \in S_{n} \mid \operatorname{sign}\left(v_{i j}^{T} r\right)=\operatorname{sign}\left(v_{n+1}^{T} r\right)\right\} & \text { if } A_{i j}=1 \\
\left\{r \in S_{n} \mid \operatorname{sign}\left(v_{i j}^{T} r\right) \neq \operatorname{sign}\left(v_{n+1}^{T} r\right)\right\} & \text { if } A_{i j}=0\end{cases}
\end{aligned}
$$

where $S_{n}$ is the surface of the unit hypersphere and $v_{i j}$ is the appropriate column of the Cholesky factor $V$ corresponding to the variable $A_{i j}$ as defined earlier. Since the only random elements of $A$ given $x_{1: K}, \rho_{1: K}$ and $Q(\bar{A})=b$ are those in $\bar{A}-A^{x \rho}$, the desired conditional distribution is given by

$$
P\left(A \mid x_{1: K}, \rho_{1: K}, Q(\bar{A})=b\right)=\frac{\operatorname{Vol}\left(\bigcap_{A_{i j} \in \bar{A}-A^{x \rho}} H\left(A_{i j}\right)\right)}{\operatorname{Vol}\left(S_{n}\right)}
$$

The expression in Eq. (26) is quite complicated since the numerator is an integral over a strange set. This is irrelevant, however, since we do not need to evaluate it. The crucial point is that samples from this distribution will approximately satisfy the prior information $Q(\bar{A})=$ $b$. Indeed, one may use the Markov inequality along with either the exact mean squared error in Eq. (17) or the bound in Eq. (23) to determine an upper bound on the probability that the squared error of a sample from this distribution will exceed any given tolerance.

\section{Approximating the Endpoint Posterior}

We use the topology and sensor ordering samples obtained in the previous section to derive an approximate endpoint posterior distribution of a passive measurement indexed by $\hat{k}$ as given in Eq. (1). If $\left\{A^{m}\right\}_{m=1}^{M}$ are the topology samples and $\left\{\rho_{1: K}^{m}\right\}_{m=1}^{M}$ are the sensor ordering samples (for each measurement), then the strong law 
of large numbers suggests a Monte Carlo estimate of the conditional expectation given by

$$
\begin{aligned}
& \hat{P}\left(u_{\hat{k}} \mid x_{1: K}, Q(\bar{A})=b\right)= \\
& \frac{1}{\kappa} \sum_{m=1}^{M} \frac{P\left(y_{\hat{k}} \mid u_{\hat{k}}, \rho_{\hat{k}}^{m}, A^{m}\right) P_{\hat{k}}\left(u_{\hat{k}}\right)}{\sum_{u} P\left(y_{\hat{k}} \mid u, \rho_{\hat{k}}^{m}, A^{m}\right) P_{\hat{k}}(u)}
\end{aligned}
$$

where $\kappa$ is a normalization constant inserted to ensure the total mass of the approximate posterior is unity. Since we are given a distribution on the endpoints of the passive measurement $P_{\hat{k}}(u)$, we need only specify a model for the conditional path probability $P(y \mid u, \rho, A)$ in order to approximate the posterior as in Eq. (27). Routing mechanisms and traffic data might figure prominently into such a model. We propose a simple model whereby the length of a path determines its probability (as in shortest path routing). If $\left|y_{\rho}\right|$ denotes the length of the ordered path $y_{\rho}$, and $y_{\rho}^{u, A}$ denotes the shortest ordered path between endpoints $u$ in topology $A$, then the conditional distribution is given by

$$
P(y \mid u, \rho, A)= \begin{cases}\theta & \text { if }\left|y_{\rho}\right|=\left|y_{\rho}^{u, A}\right|<\infty \\ 1-\theta & \text { if }\left|y_{\rho}^{u, A}\right|<\left|y_{\rho}\right|<\infty \\ 0 & \text { if }\left|y_{\rho}\right|=\infty\end{cases}
$$

The model basically says that the shortest path between endpoints $u$ in topology $A$ is chosen with probability $\theta$, and all other valid paths (that is, paths of finite length) have probability $1-\theta$. If a path does not connect the endpoints $u$ in the given topology $A$, then naturally it has zero probability. Note that for arbitrary $\theta$, we need to run Dijkstra's algorithm (or some other shortest path routing algorithm) for each topology sample $A^{m}$ in order to compute the conditional path probability in Eq. (28) [1]. This is not necessary, however, in the case that $\theta=\frac{1}{2}$ where all valid paths are equally likely.

We may give maximum a posteriori (MAP) estimates of the endpoints $u_{\hat{k}}$ of a passive measurement $y_{\hat{k}}$ after computing the posterior distribution estimate in Eq. (27). Indeed, the MAP estimate is simply given by

$$
\hat{u}_{\hat{k}}=\underset{u}{\arg \max } \hat{P}\left(u \mid x_{1: K}, Q(\bar{A})=b\right)
$$

Recall that $u \equiv(s, d)$, thus MAP estimates of $s_{\hat{k}}$ or $d_{\hat{k}}$ individually may be obtained by maximizing the appropriate marginal $\hat{P}\left(s \mid x_{1: K}, Q(\bar{A})=b\right)$ or $\hat{P}\left(d \mid x_{1: K}, Q(\bar{A})=b\right)$ respectively.

We use as an error measure the ratio $\Lambda_{u}(\hat{k})$ below for the estimated endpoints $\hat{u}_{\hat{k}}$.

$$
\Lambda_{u}(\hat{k})=\frac{\hat{P}\left(\hat{u}_{\hat{k}} \mid x_{1: K}, Q(\bar{A})=b\right)}{\hat{P}\left(\hat{u}_{\hat{k}} \mid x_{1: K}, Q(\bar{A})=b\right)+\max _{u \neq \hat{u}_{\hat{k}}} \hat{P}\left(u \mid x_{1: K}, Q(\bar{A})=b\right)}
$$

It is also useful to compute the corresponding ratios associated with the marginalized distributions $\Lambda_{s}(\hat{k})$ and $\Lambda_{d}(\hat{k})$, as it may be the case that either the source or destination of a passive measurement is more accurately determined individually than are both collectively. These are defined exactly as in Eq. (30), except $u$ is replaced with $s$ or $d$ throughout (so that the appropriate marginal distribution is considered). It is clear that the ratio in Eq. (30) must lie in the interval $\left[\frac{1}{2}, 1\right]$. Larger values of this ratio in a sense indicates more 'confidence' in the MAP estimate since a value of 1 is achieved only when all of the mass of the estimated posterior distribution is concentrated at the MAP estimate.

\section{Algorithm Complexity}

We now analyze the complexity of the source/destination estimation scheme developed here. The two fundamental quantities that determine the size of the problem are denoted by $N$ and $h ; N$ is the total number of sensors plus probing sites, so that $N=|\Gamma|+|\Sigma \cup \Delta|$, while $h$ is the maximum number of activated sensors in any measurement, so that $\left|y_{k}\right| \leq h$ for all $k=1,2, \ldots, K$. The maximum number of hops $h$ may be a function of $N$, depending upon the type of network considered. For networks that obey the small world effect, as many real world networks do, $h$ will remain approximately constant with increasing $N$ [28], [19]. The number of measurements $K$ and the number of Monte Carlo samples $M$ also affect the complexity; however we shall see the complexity dependence on these is always linear.

First note that we must store the ordering distributions $P_{k}(\rho)$ for all measurements. Since each distribution is defined over $O(h !)$ orderings, this requires $O(K h !)$ space. The adjacency matrix $A$ considers all logical connections among sensors and probing sites, so that $A$ has $O\left(N^{2}\right)$ elements. In the worst case, the linear prior information $Q(\bar{A})=b$ will constrain all elements of this matrix so that $\bar{A}=A$. It will therefore take $O\left(K N^{2} h\right.$ !) time to compute $P\left(A_{i j} \in A^{x \rho}\right)$ for all $A_{i j} \in \bar{A}$. Now in the worst case, thresholding these probabilities will produce a negligible reduction in the size of the system $Q(\bar{A})=b$, so that we still have to contend with $O\left(N^{2}\right)$ variables in solving the SDP relaxation. Typically interior point methods are used to solve SDP's to within $\epsilon$ of the optimal solution. These are based on Newton's method; therefore at each iteration it is necessary to solve a linear system of equations for the Newton directions $\left(O\left(n^{3}\right)\right.$ for a system of size $n$ ). An algorithm given in [29] is shown to take $O(|\log \epsilon| \sqrt{n})$ iterations for a problem of size $n$-this performance is typical for all interior point algorithms. Our problem has dimension $O\left(N^{2}\right)$, thus solving the SDP takes $O\left(\left(N^{2}\right)^{3.5}\right)$ or $O\left(N^{7}\right)$ time. A Cholesky factorization is then performed on the SDP solution, which takes $O\left(\left(N^{2}\right)^{3}\right)$ or $O\left(N^{6}\right)$ time.

After solving the SDP, the $M$ topology and ordering samples may be produced relatively quickly. For each 
sample, we need to draw an ordering for each of the $K$ measurements, thus requiring $O(M K)$ time to produce the ordering samples. Given the $K$ orderings for a single sample, $A^{x \rho}$ may be generated in $O(K h)$ time. Finally, we may draw the vector $r$ and take inner products to determine the remaining elements of the topology sample. Since the time required for each inner product is linear, it takes a total of $O\left(M N^{2}+M K h\right)$ time to produce the $M$ topology samples.

The final step is to compute the Monte Carlo approximation of the endpoint posterior distribution of a passive measurement. A quick inspection of Eq. (27) reveals that we need to determine the conditional path probabilities $P(y \mid u, \rho, A)$ for every endpoint pair $u-$ there are $O\left(N^{2}\right)$ such pairs. Also, computing each path probability for a given ordered path $y_{\rho}$ requires tracing this path through the topology $A$, which takes $O(h)$ time. Now, if $\theta \neq \frac{1}{2}$ we must take $O\left(M N^{3}\right)$ time to run a shortest path algorithm on each sample [1]. Therefore, it takes $O\left(M N^{2} h+M N^{3}\right)$ to produce the approximate endpoint posterior for $\theta \neq \frac{1}{2}$; this reduces to $O\left(M N^{2} h\right)$ for $\theta=\frac{1}{2}$.

The factors that give some cause for concern in this algorithm are the $h$ ! in considering all possible orderings and the $N^{7}$ in the SDP solution complexity. If we are dealing with small world networks, then $h$ might be around four or five so that $h$ ! is still manageable. And if this is not the case, one would hope that the ordering distributions $P_{k}(\rho)$ are nonzero only over a reasonable number of orderings since we need only consider $\rho$ with $P_{k}(\rho)>0$. In practice, the actual SDP complexity is likely to be significantly less than the worst case bound of $O\left(N^{7}\right)$ after reducing the system $Q(\bar{A})=b$, especially if the original prior only constrains some small subset of the adjacency elements. Our algorithm would still benefit from speedy SDP algorithms as solving the relaxation takes the most time in the worst case. A parallel implementation of an interior point algorithm for SDP's might reduce the time requirements if multiple processors are available [30].

\section{Simulations}

We performed some numerical simulations to demonstrate the utility of the method described in this paper. We generated undirected random graphs with 25 nodes to serve as test networks. The number of edges in each graph was fixed at 40 by randomly selecting 40 of the possible 300 vertex pairs and connecting the selected pairs by an edge. The adjacency matrix sparsity patterns for three example graphs are shown in Fig. 4. We randomly chose 12 of the 25 nodes to serve as probing sites-this set was then partitioned in half so that both the source set $\Sigma$ and destination set $\Delta$ each had 6 distinct elements. Sensors were placed on links in the network for two cases: $100 \%$ sensor coverage (in which all 40 links were monitored by a sensor) and $75 \%$ sensor coverage (in which 30 of the 40 links were selected at random for hosting a sensor). In the $75 \%$ coverage case, networks were generated in a rejection sampling manner so that every measurement (whether passive or active) activated at least one sensor.

In order to probe a network, we randomly selected 18 of the 36 distinct pairs in $\Sigma \times \Delta$ to serve as endpoints for active measurements. This set of 18 endpoint pairs is denoted $L \subset \Sigma \times \Delta$; the remaining pairs are denoted by $L^{c} \equiv \Sigma \times \Delta-L$. Sensor activations in response to transmissions between all pairs in $\Sigma \times \Delta$ were observed in the monitoring phase. All transmissions were routed through the network using shortest path routing, and activated sensor sets $y_{k}$ were observed. Thus for each network we had $K=54$ data points: $K_{o}-1=18$ active measurements $x_{1: 18} \equiv\left(u_{1: 18}, y_{1: 18}\right)$ and 36 passive measurements $x_{19: 54} \equiv y_{19: 54}$. For each data point ( $k=$ $1,2, \ldots K)$, a distribution on the order in which sensors were activated $P_{k}(\rho)$ was generated as follows: first the true ordering of sensors $\rho_{k}$ was noted, then noise $n(\rho)$ was drawn independently from the Uniform $[0,0.2]$ distribution for $\rho=1,2, \ldots\left|y_{k}\right|$ !, finally the distribution $P_{k}(\rho)$ was generated by normalizing the corrupted delta function distribution as in Eq. (31).

$$
P_{k}(\rho)=\frac{\delta\left(\rho-\rho_{k}\right)+n(\rho)}{\sum_{\rho=1}^{\left|y_{k}\right| !} \delta\left(\rho-\rho_{k}\right)+n(\rho)}
$$

The linear prior information was generated from degree information on the logical topology $A$. Indeed vertex degree information is a commonly used special case of the more general linear prior specified by $Q(\bar{A})=b$ [20], [23]. The sensor degree, that is the number of sensors $b_{i}$ to which the $i^{t h}$ vertex in the logical topology is adjacent, was known for all $v_{i} \in V_{A}$. In addition to knowing the sensor degrees of vertices in the logical topology $G_{A}$, a random subset consisting of no more than $60 \%$ of the sensors not adjacent to a given vertex were also known. For the $i^{t h}$ vertex, the $i^{t h}$ row of the operator $Q_{i}(\bar{A})$ therefore sums over the elements of $A$ for which adjacency to vertex $i$ is uncertain, and the $i^{t h}$ element of $b, b_{i}$, is simply the known sensor degree of vertex $i$. As an example, consider vertex $\gamma_{5}$ of the logical topology in Fig. 3. Vertex $\gamma_{5}$ is adjacent to sensors $\left\{\gamma_{2}, \gamma_{4}\right\}$, therefore its sensor degree is two. Since there are two sensors not adjacent to $\gamma_{5},\lfloor 2 * 60 \%\rfloor=1$ sensor, say $\gamma_{x}$, is selected at random from the set $\left\{\gamma_{1}, \gamma_{3}\right\}$. The row of the prior $Q(\bar{A})=b$ corresponding to $\gamma_{5}$ is then given by $\sum_{j \in \Gamma-\gamma_{5}-\gamma_{x}} A_{\gamma_{5} j}=2$.

Given the sensor degree prior information and the ordering distributions, we eliminated those adjacency elements whose probability of being in the set $A^{x \rho}$ exceeded $\frac{1}{2}$ from $\bar{A}$, where $P\left(A_{i j} \in A^{x \rho}\right)$ was computed 


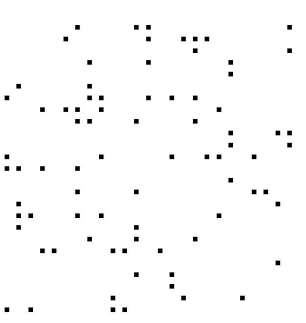

A

Fig. 4. Example sparsity patterns for the adjacency matrices of three undirected random graphs with 25 nodes and 40 randomly selected links. The method is illustrated by simulating on topologies of this type. 12 of the 25 nodes are selected as probing sites: 6 of these are taken as sources $\Sigma$ and 6 are taken as destinations $\Delta$. We assume in one case that sensors are placed on all 40 links (100\% sensor coverage) and in another that sensors are placed on 30 randomly selected links (75\% sensor coverage). Active measurements consist of 18 of the 36 distinct pairs in $\Sigma \times \Delta$ randomly selected for use in the probing phase, denoted $L$. The remaining 18 pairs are denoted $L^{c}$. Passive measurements consist of sensor activations monitored between all pairs in $\Sigma \times \Delta$. Shortest path routing is used to determine the transmission path.

as in Eq. (24). The reduced system $Q(\bar{A})=b$ was then used to formulate the SDP relaxation in Eq. (13) for the minimum norm solution with the weight matrix $\Lambda$ taken as the identity. The relaxation was solved with a predictor-corrector path following algorithm given in [4]. A publicly available $\mathrm{C}$ implementation of this algorithm was used [31]. The SDP solution was used along with the ordering distributions $P_{k}(\rho)$ to produce $M=500$ samples of measurement orderings $\rho_{1: K}$ and adjacency matrices $A$ for computing the Monte Carlo estimates of the endpoint posteriors.

We assumed the endpoint priors $P_{k}(u)$ were uniform over $\Sigma \times \Delta$ for all 36 passive measurements $k=$ $19,20, \ldots, 54$. Also, the parameter $\theta$ in the conditional path probabilities of Eq. (28) was taken as $\frac{1}{2}$ so that it was not necessary to run a shortest path routing algorithm on every sample topology. The 500 ordering and topology samples were then used to compute the approximate endpoint posteriors for all passive measurements as given in Eq. (27). These were used to produce joint MAP estimates of the transmission endpoints and to compute the resolution measures $\Lambda_{u}(\hat{k})$. An example endpoint posterior is given in Fig. 5, for which the correct endpoint pair is source no. 6 and destination no. 3. It is clear that the MAP estimate will result in the correct pair in this case. Also indicated in the Figure is the second most likely pair $u=(6,5)$; this is used in computing the resolution measure $\Lambda_{u}$ as in Eq. (30)$\Lambda_{u}(\hat{k})=0.60$ for this case. Marginal distributions of the approximate posterior are given in Fig. 6. These were used in individual MAP estimation of source and destination. It is clear that the individual estimates will match the joint estimate for this case; the resolution measures were a bit lower though with $\Lambda_{s}(\hat{k})=0.58$ and $\Lambda_{d}(\hat{k})=0.59$. This completes the simulation process for a single graph.

We repeated the simulation procedure for 30 net- works with $100 \%$ sensor coverage and 30 networks with $75 \%$ sensor coverage. Table I demonstrates the effectiveness of the SDP randomized rounding algorithm for producing topology samples that approximately agree with the sensor degree prior information. It lists the normalized squared topology sample error $\frac{1}{\|Q e\|^{2}+\|b\|^{2}} \frac{1}{M} \sum_{m}\left\|Q \hat{a}^{m}-b\right\|^{2}$ averaged over the $M=500$ samples along with the normalized expected squared error $\frac{1}{\|Q e\|^{2}+\|b\|^{2}} E\left[\|Q \hat{a}-b\|^{2}\right]$ as in Eq. (17) for each graph. The bound derived in Eq. (23) assures the expected squared error can never exceed $1-\alpha \approx 0.12$. We see that graphs with $75 \%$ sensor coverage tend to have lower error values.

Plots of proportion of passive measurement endpoint estimates correct for a given set $\left(L\right.$ or $\left.L^{c}\right)$ versus the resolution ratio from Eq. (30) averaged over the corresponding set are given in Fig. 7. Plots are shown for joint estimates of $u_{\hat{k}}$ via the joint distribution as well as for individual estimates of $s_{\hat{k}}$ and $d_{\hat{k}}$ from the marginals. We observe an approximately linear relation between the proportion of correct estimates and the appropriate $\Lambda$ ratio when the $\Lambda$ ratio exceeds 0.68 . In this regime, the $\Lambda$ ratio might be used as a measure of confidence in the endpoint estimates. Also note that transmissions in set $L$ tend to have higher $\Lambda$ ratios (and are correct more often) than those in set $L^{c}$ because it is the transmissions in set $L$ that are used in training the probing sites. We see that marginalized MAP estimates are often better than joint MAP estimates. Marginalization certainly blurs the linear relation in the higher confidence regime. We also observe some degradation in the quality of the estimates when only $75 \%$ of the links are equipped with sensors; this is to be expected though. Recall that these results are obtained with completely random placement of sensors and random choices for the $(s, d)$ pairs to use in the probing phase. These two factors will clearly affect 


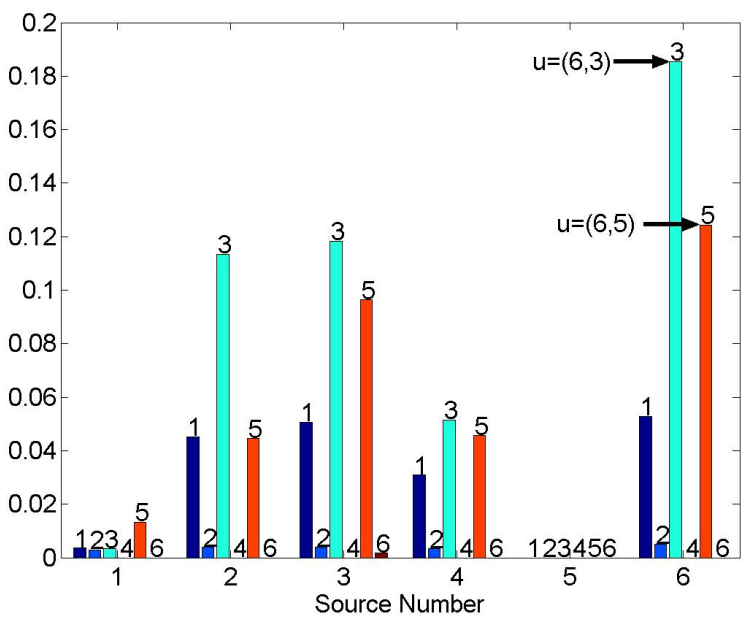

A

Fig. 5. Example endpoint posterior distribution $\hat{P}\left(u_{\hat{k}} \mid x_{1: K}, Q(\bar{A})=b\right)$ for a passive measurement $\hat{k}$ with endpoints $u=$ $(s, d)=(6,3)$. In plot $\mathrm{A}$, the probabilities are grouped by source, with each of 6 bars in a group corresponding to a different destination (noted above the individual bar). Plot B displays the same information except probabilities are grouped by destination with source number noted above each individual bar. The largest and second largest values of the posterior are indicated-it is these values that are used in computing the resolution ratio $\Lambda_{u}$ of Eq. (30), calculated as $\Lambda_{u}(\hat{k})=0.60$. It is clear in this example that the endpoints of this transmission will be correctly estimated by the joint MAP estimate.

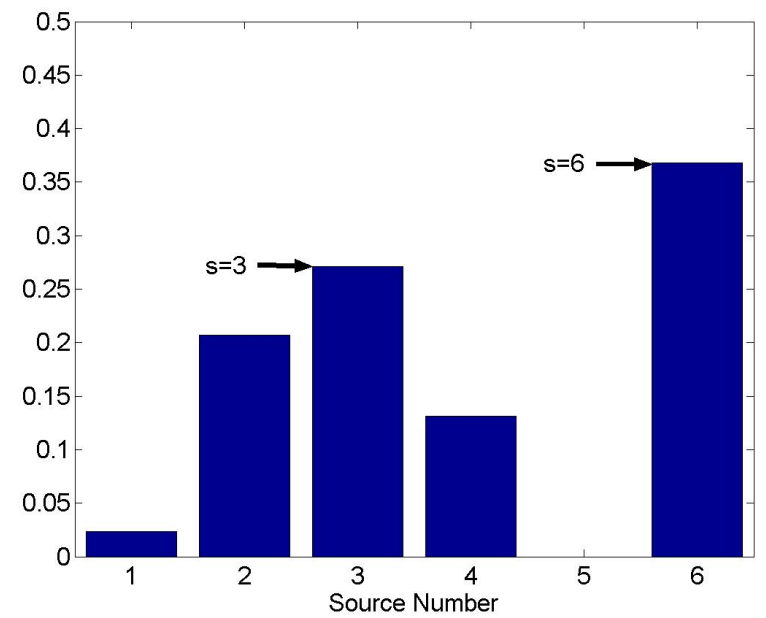

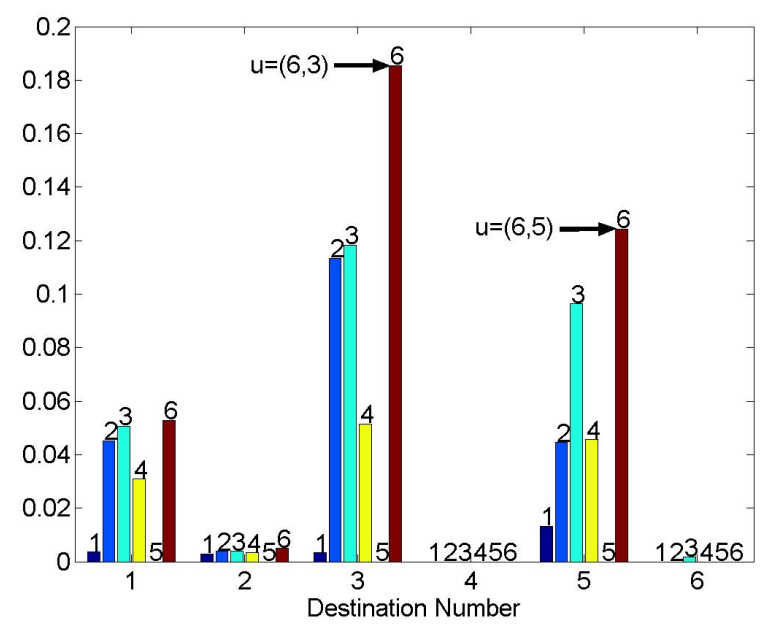

B

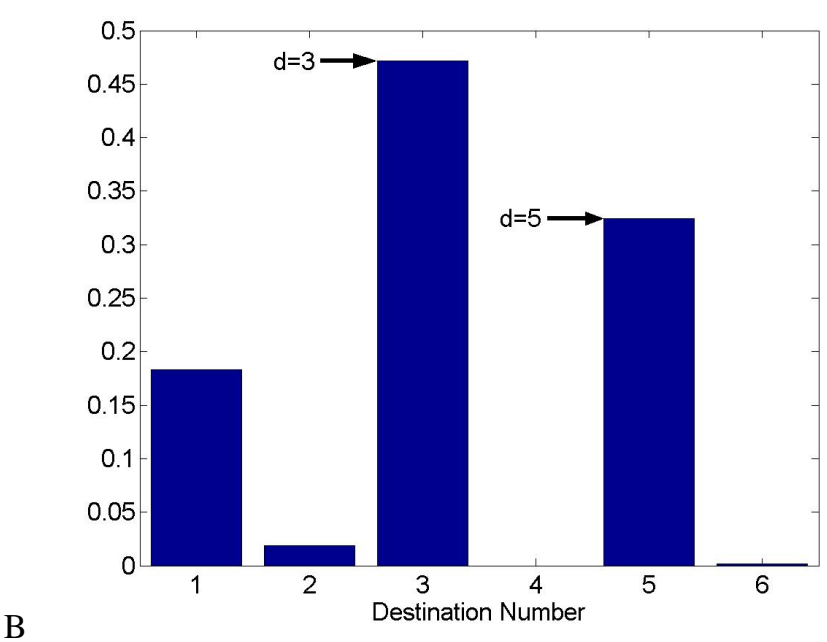

Fig. 6. Marginal distributions $\left(\hat{P}\left(s_{\hat{k}} \mid x_{1: K}, Q(\bar{A})=b\right)\right.$ in A and $\hat{P}\left(d_{\hat{k}} \mid x_{1: K}, Q(\bar{A})=b\right)$ in $\left.\mathrm{B}\right)$ associated with the example endpoint posterior distribution shown in Fig. 5. The largest and second largest values of the marginal posteriors are indicated-it is these values that are used in computing the resolution ratios $\Lambda_{s}$ and $\Lambda_{d}$, calculated as $\Lambda_{s}(\hat{k})=0.58$ and $\Lambda_{d}(\hat{k})=0.59$. It is clear in this example that the endpoints of this transmission (source number 6 and destination number 3 ) will be correctly estimated by the individual MAP estimates as well.

the estimates of passive measurement endpoints, and therefore provide an interesting direction for future work.

\section{SUMMARY AND EXTENSIONS}

In this paper, we have developed a methodology for estimating the endpoints of a transmission in a network using link-level transmission interceptions. The estimation is done using Monte Carlo simulation in a Bayesian framework. A semidefinite programming relaxation is used to generate logical network topology samples that approximately agree with linear prior information. It is possible to envision applications of the method in all sorts of networks, or systems with key features modeled by networks. We have displayed simulations of its utility on some random networks. We now discuss some extensions of the theory presented here and possibilities for 


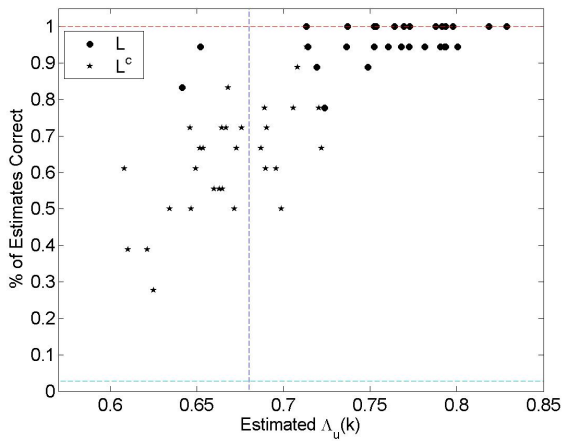

$\Lambda_{u}, 100 \%$ coverage

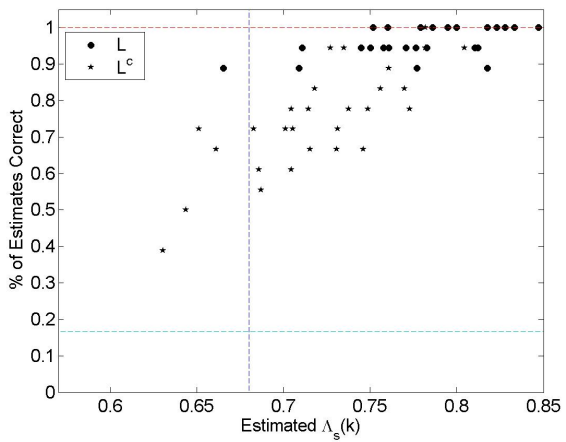

$\Lambda_{s}, 100 \%$ coverage

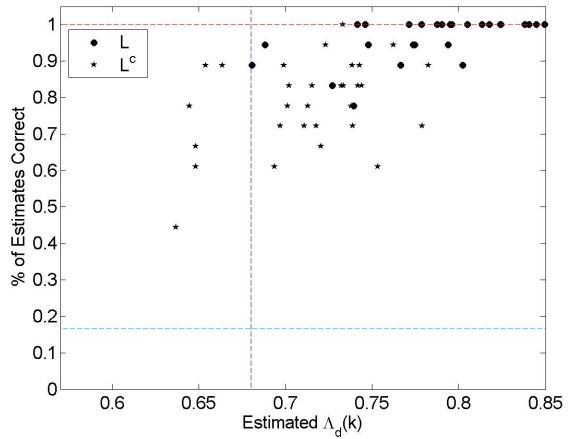

$\Lambda_{d}, 100 \%$ coverage

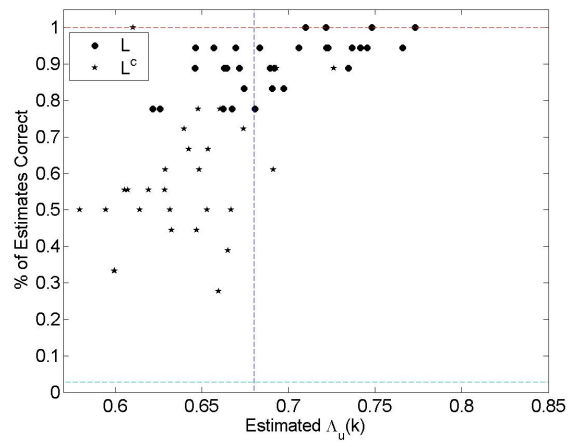

$\Lambda_{u}, 75 \%$ coverage

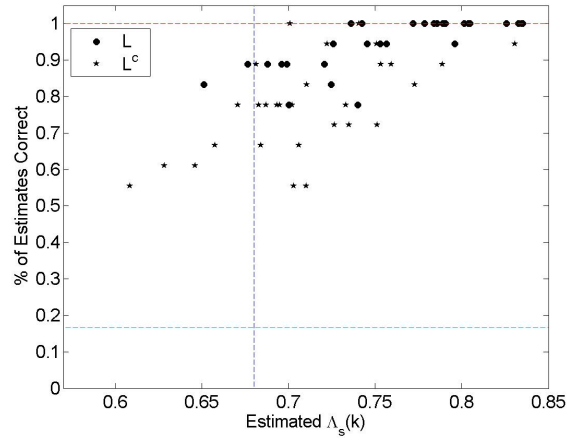

$\Lambda_{s}, 75 \%$ coverage

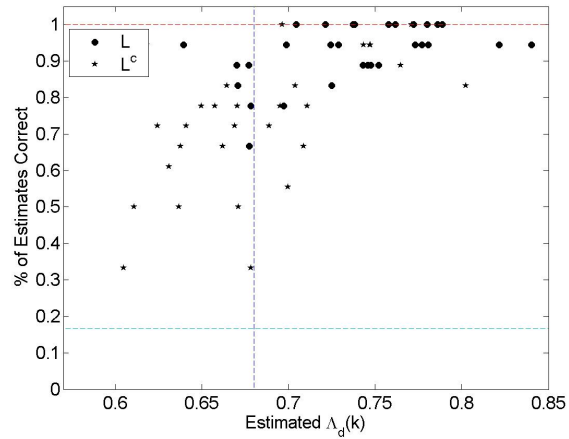

$\Lambda_{d}, 75 \%$ coverage

Fig. 7. Plots of proportion of endpoint estimates correct for a given set ( $L$ or $L^{c}$ ) versus the resolution ratios of Eq. (30) averaged over the corresponding set for the two simulation cases: $100 \%$ sensor coverage in the first column and $75 \%$ sensor coverage in the second. Circles indicate averages over paths from set $L$ and pentagrams indicate averages over paths from set $L^{c}$. The first row $\left(\Lambda_{u}\right)$ is for joint MAP estimation of $u_{\hat{k}}=\left(s_{\hat{k}}, d_{\hat{k}}\right)$ from joint distribution $\hat{P}\left(u_{\hat{k}} \mid x_{1: K}, Q(\bar{A})=b\right)$. The second row $\left(\Lambda_{s}\right)$ is for individual estimation of $s_{\hat{k}}$ from marginal distribution $\hat{P}\left(s_{\hat{k}} \mid x_{1: K}, Q(\bar{A})=b\right)$. The third row $\left(\Lambda_{d}\right)$ is for individual estimation of $d_{\hat{k}}$ from marginal distribution $\hat{P}\left(d_{\hat{k}} \mid x_{1: K}, Q(\bar{A})=b\right)$. Some reference lines are also plotted. The chance line for randomly selecting endpoints is drawn in each plot (1/36 for joint estimation and $1 / 6$ for individual estimation). Note that above $\Lambda(\hat{k})=0.68$, an approximately linear behavior is observed. This behavior is somewhat washed out for the marginalized estimates, however marginalizing tends to increase the percent of correct estimates. It is not surprising that there appears to be some degradation in the quality of the estimates when only $75 \%$ of the links are equipped with sensors. 


\begin{tabular}{|c|c|c|c|}
\hline $100 \%$ Avg & $100 \%$ Exp & $75 \%$ Avg & $75 \%$ Exp \\
\hline 0.0211 & 0.0208 & 0.0142 & 0.0145 \\
0.0229 & 0.0214 & 0.0110 & 0.0113 \\
0.0195 & 0.0200 & 0.0108 & 0.0111 \\
0.0247 & 0.0241 & 0.0119 & 0.0117 \\
0.0178 & 0.0170 & 0.0146 & 0.0146 \\
0.0247 & 0.0257 & 0.0152 & 0.0156 \\
0.0189 & 0.0200 & 0.0139 & 0.0133 \\
0.0247 & 0.0236 & 0.0154 & 0.0155 \\
0.0230 & 0.0221 & 0.0143 & 0.0138 \\
0.0222 & 0.0221 & 0.0121 & 0.0123 \\
0.0243 & 0.0244 & 0.0135 & 0.0141 \\
0.0241 & 0.0229 & 0.0118 & 0.0117 \\
0.0217 & 0.0209 & 0.0139 & 0.0136 \\
0.0190 & 0.0182 & 0.0125 & 0.0125 \\
0.0248 & 0.0235 & 0.0127 & 0.0125 \\
0.0195 & 0.0198 & 0.0131 & 0.0140 \\
0.0257 & 0.0261 & 0.0133 & 0.0138 \\
0.0180 & 0.0182 & 0.0147 & 0.0143 \\
0.0236 & 0.0237 & 0.0137 & 0.0131 \\
0.0214 & 0.0213 & 0.0122 & 0.0113 \\
0.0250 & 0.0237 & 0.0112 & 0.0117 \\
0.0253 & 0.0255 & 0.0128 & 0.0119 \\
0.0191 & 0.0207 & 0.0135 & 0.0139 \\
0.0186 & 0.0196 & 0.0150 & 0.0142 \\
0.0200 & 0.0219 & 0.0142 & 0.0140 \\
0.0272 & 0.0245 & 0.0119 & 0.0122 \\
0.0212 & 0.0221 & 0.0110 & 0.0109 \\
0.0188 & 0.0188 & 0.0112 & 0.0114 \\
0.0244 & 0.0249 & 0.0128 & 0.0130 \\
0.0269 & 0.0269 & 0.0116 & 0.0110 \\
\hline
\end{tabular}

TABLE I

SQUARED ERROR VALUES FOR COMPLIANCE OF SAMPLES WITH LINEAR PRIOR INFORMATION $Q(\bar{A})=b$. SAMPLE TOPOLOGY ERRORS (AVG) AVERAGED OVER THE 500

SAMPLES PRODUCED FOR EACH OF THE THIRTY GRAPHS IN THE TWO SIMULATION CASES (100\% COVERAGE AND 75\% COVERAGE) ARE GIVEN ALONG WITH THE THEORETICAL EXPECTED VALUE OF THE ERROR (EXP). ONCE THE ELEMENTS OF $\bar{A}$ ARE ORGANIZED IN THE VECTOR $a$, THE NORMALIZED AVERAGE SAMPLE ERROR (AVG) IS SIMPLY $\frac{1}{\|Q e\|^{2}+\|b\|^{2}} \frac{1}{M} \sum_{m}\left\|Q \hat{a}^{m}-b\right\|^{2}$ FOR THE $m^{t h}$ SAMPLE $\hat{a}^{m}$ PRODUCED BY THE SDP ROUNDING METHOD. THE NORMALIZED EXPECTED ERROR (EXP) $\frac{1}{\|Q e\|^{2}+\|b\|^{2}} E\left[\|Q \hat{a}-b\|^{2}\right]$ AS DERIVED IN EQ. (17) IS ALSO GIVEN. NOTE THAT THE BOUND IN EQ. (23) ASSURES (EXP) NEVER EXCEEDS $1-\alpha \approx 0.12$. WE SEE ALSO THAT THE GRAPHS WITH 75\% SENSOR COVERAGE TYPICALLY HAVE LOWER SQUARED ERROR VALUES. future work on this problem.

It is possible to extend our algorithm for source/destination estimation to the cases of noisy sensors and sensor excitation due to multiple transmissions without much trouble. Consider first when the sensors are noisy: then the observed set of activated sensors $y$ may not match the true set of sensors $\tilde{y}$ passed by a particular transmission. Suppose that each sensor $\gamma \in \Gamma$ has an associated miss probability $\alpha_{m}(\gamma)=P(\gamma \notin y \mid \gamma \in \tilde{y})$ and false alarm probability $\alpha_{f}(\gamma)=P(\gamma \in y \mid \gamma \notin \tilde{y})$. The probing mechanism then repeats the data transmission from $\sigma_{k}$ to $\delta_{k} N$ times for each $k$. These $N$ measurements are used to construct a maximum likelihood estimate $\hat{y}_{k}$ of each path $\tilde{y}_{k}$ according to the following model. Along the lines of a generalized likelihood approach, the measurement mechanism passes along the maximum likelihood path estimates for each $\tilde{y}_{k}$ for use in approximating the endpoint posterior. Note that we will likely have to settle for $N=1$ for passive measurements.

Define the path indicator vector $\nu$ whose elements are given by $\nu(j)=I_{y}\left(\gamma_{j}\right)$ for all $j=1,2, \ldots|\Gamma|$ where $I_{A}: A \rightarrow\{0,1\}$ is the usual indicator function. If we assume sensor errors are independent across paths and measurements, then the joint probability mass function of the $N$ observed path vectors for a given source/destination pair $\nu_{i}$ is

$$
\begin{aligned}
& P\left(\nu_{1}, \nu_{2}, \ldots \nu_{N} \mid \tilde{\nu}\right)= \\
& \prod_{k=1}^{N} \prod_{j=1}^{|\Gamma|} \alpha_{m}\left(\gamma_{j}\right)^{\left(1-\nu_{k}(j)\right) \tilde{\nu}(j)} \beta_{m}\left(\gamma_{j}\right)^{\nu_{k}(j) \tilde{\nu}(j)} \\
& \alpha_{f}\left(\gamma_{j}\right)^{\nu_{k}(j)(1-\tilde{\nu}(j))} \beta_{f}\left(\gamma_{j}\right)^{\left(1-\nu_{k}(j)\right)(1-\tilde{\nu}(j))}
\end{aligned}
$$

where $\beta_{m}(\gamma) \equiv 1-\alpha_{m}(\gamma)$ and $\beta_{f}(\gamma) \equiv 1-\alpha_{f}(\gamma)$. If we define the likelihood function $L(\tilde{\nu})$ as the logarithm of the expression in Eq. (32), then it may be written explicitly as

$$
\begin{aligned}
& L(\tilde{\nu})= \\
& \sum_{j=1}^{|\Gamma|}\left(N \log \beta_{f}\left(\gamma_{j}\right)+\sum_{k=1}^{N} \nu_{k}\left(\gamma_{j}\right) \log \frac{\alpha_{f}\left(\gamma_{j}\right)}{\beta_{f}\left(\gamma_{j}\right)}\right)+ \\
& \sum_{j=1}^{|\Gamma|}\left(N \log \frac{\alpha_{m}\left(\gamma_{j}\right)}{\beta_{f}\left(\gamma_{j}\right)}+\sum_{k=1}^{N} \nu_{k}\left(\gamma_{j}\right) \log \frac{\beta_{e}\left(\gamma_{j}\right)}{\alpha_{e}\left(\gamma_{j}\right)}\right) \tilde{\nu}\left(\gamma_{j}\right)
\end{aligned}
$$

where $\alpha_{e}(\gamma) \equiv \alpha_{m}(\gamma) \alpha_{f}(\gamma)$ and $\beta_{e}(\gamma) \equiv \beta_{m}(\gamma) \beta_{f}(\gamma)$. Since only the second term in Eq. (33) depends on $\tilde{\nu}$ and $\tilde{\nu} \in\{0,1\}^{|\Gamma|}$, the maximum likelihood path estimate may be written quite compactly as

$$
\begin{aligned}
& \hat{y}= \\
& \left\{\gamma_{j} \in \Gamma \mid N \log \frac{\alpha_{m}\left(\gamma_{j}\right)}{\beta_{f}\left(\gamma_{j}\right)}+\sum_{k=1}^{N} \nu_{k}(j) \log \frac{\beta_{e}\left(\gamma_{j}\right)}{\alpha_{e}\left(\gamma_{j}\right)} \geq 0\right.
\end{aligned}
$$

As another extension, suppose that for passive measurements the activated sensor set $y_{k}$ is due to transmissions passed between $n$ source/destination pairs $u_{k i}$ for $i=1,2, \ldots, n$ instead of just a single pair. The strategy here is to introduce a random variable $\eta_{k}$ for each passive 
measurement that represents a partition of the activated sensor set $y_{k}$ into sets $y_{k i}$ for $i=1,2, \ldots, n$, where the sensors in each $y_{k i}$ are activated in response to a single transmission. We may then split the single measurement $y_{k}$ into $n$ different passive measurements $y_{k i}$ according to the value of the partition variable $\eta_{k}$ and proceed with the previous theoretical development. In this case, the endpoint posterior of Eq. (1) becomes

$$
\begin{aligned}
& P\left(u_{\hat{k}} \mid x_{1: K}, Q(\bar{A})=b\right)= \\
& E_{A, \rho_{1: K}, \eta_{K_{o}: K}}\left[\frac{P\left(y_{\hat{k}} \mid u_{\hat{k}}, \rho_{\hat{k}}, A\right) P_{\hat{k}}\left(u_{\hat{k}}\right)}{\sum_{u} P\left(y_{\hat{k}} \mid u, \rho_{\hat{k}}, A\right) P_{\hat{k}}(u)} \mid x_{1: K}, Q(\bar{A})=b\right]
\end{aligned}
$$

where we must now also take the expectation over partition variables $\eta_{K_{o}: K}$ of all passive measurements. The first step of the Monte Carlo sampling would then be to draw a partition variable for each passive measurement from some (presumably available) distribution $P_{k}(\eta)$. Given the partition variable, appropriate orderings may be drawn and so on as before.

One can similarly account for the case of random linear prior information $Q(\bar{A})=b$. Suppose that instead of being given a fixed operator $Q$ and vector $b$, we are given a distribution on these $P(Q, b)$. This might occur, for example, when we know that the vertex degrees follow a power-law distribution [22]- in which case a distribution on $b$ is induced. We must now also take the expectation over $Q$ and $b$, so that the endpoint posterior becomes

$$
\begin{aligned}
& P\left(u_{\hat{k}} \mid x_{1: K}\right)= \\
& E_{A, \rho_{1: K}, Q, b}\left[\frac{P\left(y_{\hat{k}} \mid u_{\hat{k}}, \rho_{\hat{k}}, A\right) P_{\hat{k}}\left(u_{\hat{k}}\right)}{\sum_{u} P\left(y_{\hat{k}} \mid u, \rho_{\hat{k}}, A\right) P_{\hat{k}}(u)} \mid x_{1: K}\right]
\end{aligned}
$$

A Monte Carlo approximation of Eq. (36) would therefore require drawing $Q$ and $b$ then proceeding as before. Unfortunately, a new SDP must be solved for every $Q$ and $b$ in order to produce topology samples $A$. If the SDP relaxation is not too large, this might be reasonable. If the size is prohibitive, one might approximate the expectation by selecting only a few of the most likely realizations of $(Q, b)$ and solving the SDP for these. The distribution $P(Q, b)$ is then restricted to be nonzero only at elements of this preselected dictionary so that the Monte Carlo simulation selects those only those values for which we have already solved the SDP.

An interesting direction for future work would be to develop an adaptive probing scheme. It is obvious that the quality of endpoint estimates for suspect transmissions will depend on which endpoints were used in the probing phase. The idea here is to use the approximate endpoint posterior distributions to suggest additional active measurements that should be made in order to improve the estimates. The diagram for such a system is shown in Fig. 8. One can hypothesize criteria for determining the new probing pairs. For example, nodes that tend to have similar posterior probabilities over several suspect paths might be selected for probing so as to distinguish them more explicitly in the constraints. The question of efficient online implementation naturally arises in this context. A forgetting factor could be used in conjunction with existing topology and ordering samples so that an entirely new batch would not be required at each probing cycle.

\section{ACKNOWLEDGEMENTS}

This work was partially supported by a Dept. of EECS Graduate Fellowship to the first author and by the National Science Foundation under ITR contract CCR0325571.

\section{REFERENCES}

[1] C. Papadimitriou and K. Steiglitz, Combinatorial Optimization: Algorithms and Complexity. Englewood Cliffs, NJ: Prentice Hall, Inc., 1982.

[2] L. Lovasz and A. Schrijver, "Cones of matrices and set-functions and 0-1 optimization," SIAM Journal on Optimization, vol. 1, no. 2, pp. 166-190, 1991.

[3] R. Saigal, Linear Programming: A Modern Integrated Analysis. Boston: Kluwer Academic Publishers, 1995.

[4] C. Helmberg, F. Rendl, R. Vanderbei, and H. Wolkowicz, "An interior-point method for semidefinite programming," SIAM $J$. Optimization, vol. 6, no. 2, pp. 342-361, May 1996.

[5] F. Alizadeh, "Interior point methods in semidefinite programming with applications to combinatorial optimization," SIAM Journal on Optimization, vol. 5, no. 1, pp. 13-51, 1995.

[6] M. Goemans and D. Williamson, "Improved approximation algorithms for maximum cut and satisfiability problems using semidefinite programming," Journal of the ACM, vol. 42, no. 6, pp. 1115-1145, Nov. 1995.

[7] M. Goemans and F. Rendl, "Semidefinite programming in combinatorial optimzation," in Handbook of Semidefinite Programming, H. Wolkowicz, R. Saigal, and L. Vandenberghe, Eds. Kluwer Academic Publishers, 2000, pp. 343-360.

[8] C. Helmberg, "Fixing variables in semidefinite relaxations," SIAM J. Matrix Anal. and Apps., vol. 21, no. 3, pp. 952-969, 2000.

[9] Y. Vardi, "Network tomography: estimating the sourcedestination traffic intensities from link data," J. Amer. Stat. Assoc., vol. 91, pp. 365-377, 1996.

[10] G. Liang and B. Yu, "Maximum pseudo likelihood estimation in network tomography," IEEE Transactions on Signal Processing, vol. 51, no. 8, pp. 2043-2053, Aug. 2003.

[11] M. Shih and A. Hero, "Unicast-based inference of network link delay distributions with finite mixture models," IEEE Transactions on Signal Processing, vol. 51, no. 8, pp. 2219-2228, Aug. 2003.

[12] Y. Tsang, M. Coates, and R. Nowak, "Network delay tomography," IEEE Transactions on Signal Processing, vol. 51, no. 8, pp. 2125-2136, Aug. 2003.

[13] R. Caceres, N. Duffield, J. Horowitz, and D. Towsley, "Multicastbased inference of network-internal loss characteristics," IEEE Transactions on Information Theory, vol. 45, no. 7, pp. 24622480, 1999.

[14] M. Coates, R. Castro, and R. Nowak, "Maximum likelihood network topology identification from edge-based unicast measurements," ACM Sigmetric 2002, June 2002.

[15] N. Duffield, J. Horowitz, F. L. Presti, and D. Towsley, "Multicast topology inference from measured end-to-end loss," IEEE Transactions on Information Theory, vol. 48, pp. 26-45, Jan 2002.

[16] M. Coates, A. Hero, R. Nowak, and B. Yu, "Internet tomography," IEEE Signal Processing Magazine, vol. 19, no. 3, pp. 47-65, May 2002. 


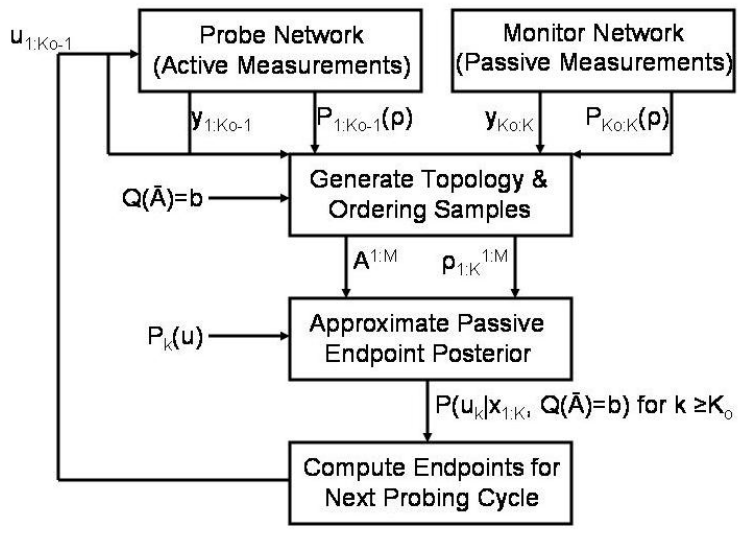

Fig. 8. Diagram of the adaptive transmission endpoint estimation system with feedback of new source/destination pairs to probe.

[17] J. Treichler, M. Larimore, S. Wood, and M. Rabbat, "Determining the topology of a telephone system using internally sensed network tomography," Proc. of 11th Digital Signal Processing Workshop, Aug. 2004.

[18] M. Rabbat and R. Nowak, "Telephone network topology inference," Univ. of Wisconsin, Madison, WI, Tech. Rep., Dec 2004.

[19] M. Newman, "The structure and function of complex networks," SIAM Review, vol. 45, pp. 167-256, 2003.

[20] Q. Morris, B. Frey, and C. Paige, "Denoising and untangling graphs using degree priors," Proc. Neural Info. Proc. Sys., no. 16, 2003.

[21] A.-L. Barabasi and R. Albert, "Emergence of scaling in random networks," Science, vol. 286, no. 5439, pp. 509-512, Oct. 1999.

[22] M. Faloutsos, P. Faloutsos, and C. Faloutsos, "On power-law relationships of the internet topology," Proc. of ACM SIGCOMM'99, pp. 251-262, Aug. 1999.

[23] S. Gomez and A. Rzhetsky, "Towards the prediction of complete protein-protein interaction networks," Proc. Pacific Symp. on Biocomputing, pp. 413-424, 2002.

[24] E. Moore, "Gedanken-experiments on sequential machines," in Automata Studies, Annals of Mathematics Studies. Princeton, N.J.: Princeton University Press, 1956, no. 34, pp. 129-153.

[25] D. Lee and M. Yannakakis, "Principles and methods of testing finite state matchines - a survey," Proc. of the IEEE, vol. 84, no. 8, pp. 1090-1123, Aug. 1996.

[26] M. Beal and Z. Ghahramani, "The variational bayesian em algorithm for incomplete data: with application to scoring graphical model structures," Bayesian Statistics, vol. 7, pp. 453-464, 2003.

[27] M. Garey and D. Johnson, Computers and Intractability: A Guide to the Theory of NP-Completeness. San Francisco, CA: W.H. Freeman, 1979.

[28] S. Milgram, "The small world problem," Psychology Today, vol. 2, pp. 60-67, 1967.

[29] C.-J. Lin and R. Saigal, "A predictor corrector method for semidefinite linear programming," Department of Industrial and Operations Engineering, University of Michigan, Ann Arbor, MI, Tech. Rep. TR95-20, Oct. 1995.

[30] M. Nayakkankuppam and Y. Tymofyeyev, "A parallel implementation of the spectral bundle method for large-scale semidefinite programs," Proc. of the 8th SIAM Conf. on App. Lin. Alg., 2003.

[31] B. Borchers, "Csdp: A c library for semidefinite programming," Optimization Methods and Software, vol. 11, no. 1, pp. 613-623, 1999. 